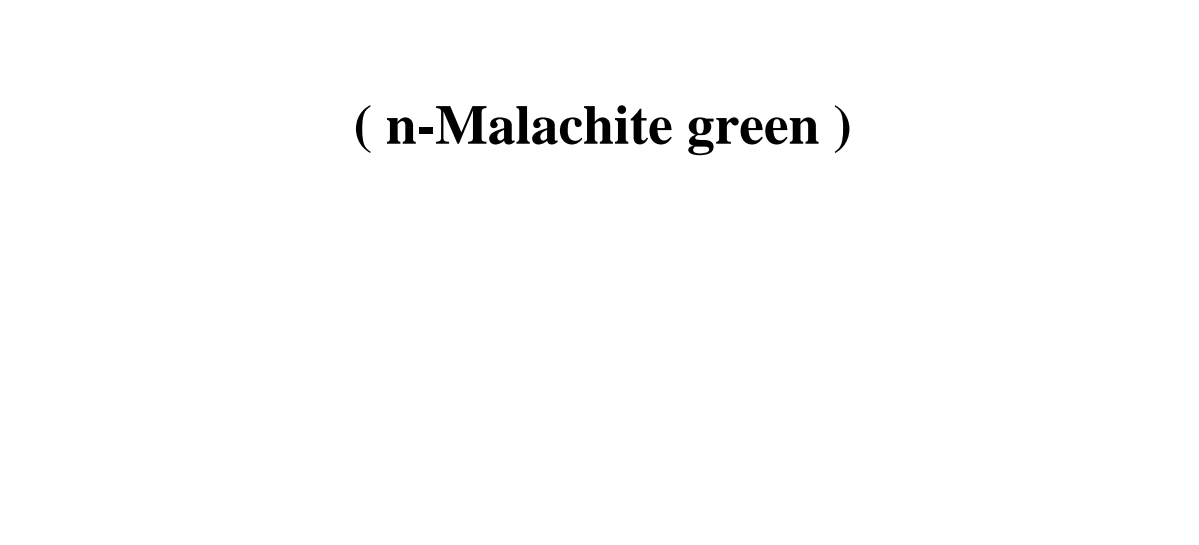

(تاريخ الاستلام 12/ 11 / 2010 ؛ تاريخ القبول 25 / 4 / 2011)

\title{
الملغص
}

في هذا البحث، فم تصنبع ثنائيشوتكي بلستخدلم مادةشبه موصلة عضوية (n-Malachite green)

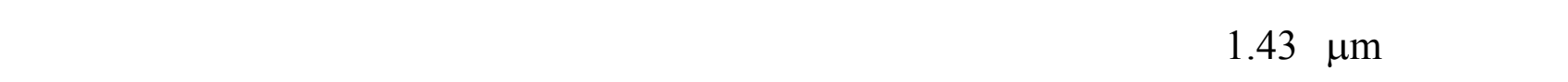

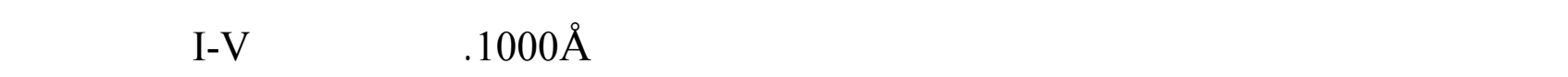

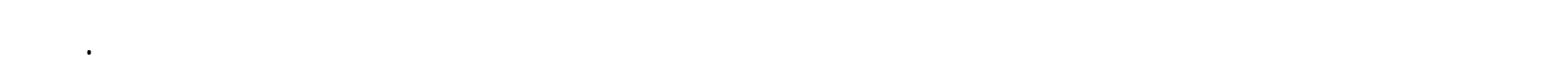

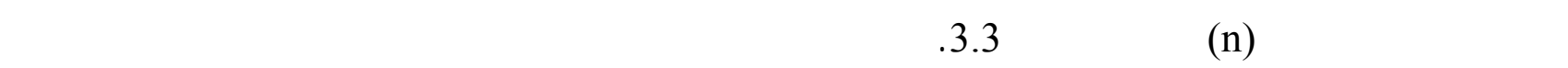

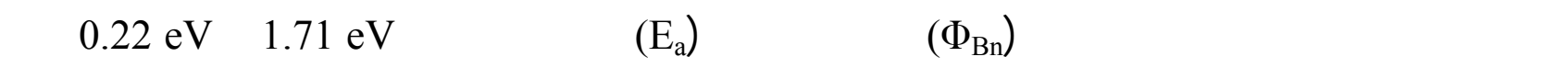

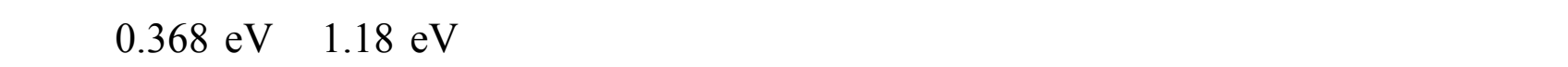

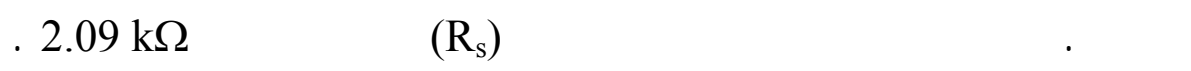
الكاملت الدالة :شبه الموصل العضوي، ثنائيشوتكي، كهربائي .

\section{Study of the Electrical Characteristics of Schottky Organic Semiconductor (n-Malachite green), Diode}

Ismail K. Abbas
Zahraa H. Ahmed

\section{ABSTRACT}

In this research, Schottky diodes were fabricated using the pure organic semiconductor $\mathrm{n}$ - malachite green with thickness $1.43 \mu \mathrm{m}$ at room temperature. The electrodes and the 
ohmic contacts were Indium and aluminum respectively. These materials were deposited by vacuum evaporation process with thickness $1000 \AA$. Current-Voltage characteristics were studied at room temperature in dark and light, and the sample showed the characteristic of schottky diode. The calculated value of ideal factor (n) is 3.3. We study the variation of conductivity with temperature in light from which the potential barrier $\left(\Phi_{\mathrm{Bn}}\right)$ and activation energy $\left(\mathrm{E}_{\mathrm{a}}\right)$ were calculated and their values are 1.71 and $0.22 \mathrm{eV}$ at room temperature respectively. But at higher temperature their values are 1.18 and $0.368 \mathrm{eV}$ respectively. Finally the series resistance $\left(R_{s}\right)$ also had been calculated and its value is 2.09 $\mathrm{k} \Omega$.

Keywords : Organic Semiconductor, schottky diode, electrical .

\section{الاقمة}

تمكن العالمانشوتكي وموت علم 1938 من المساهمة في قطوير الصناعلت الألكترونية وذل لـ مـ ـن

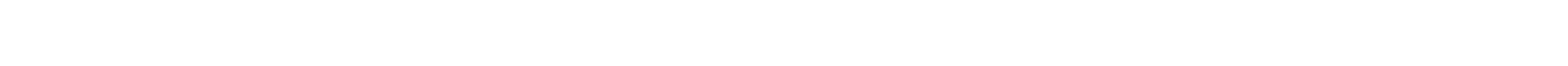

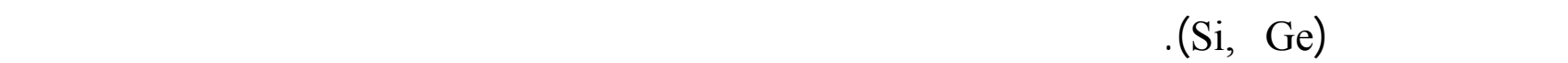
اللاعضوية الاعتيادية بلثبا الموصلات العضوية في الاجهزة الكهربيائية والبصرية ( Seyd Abthagir and

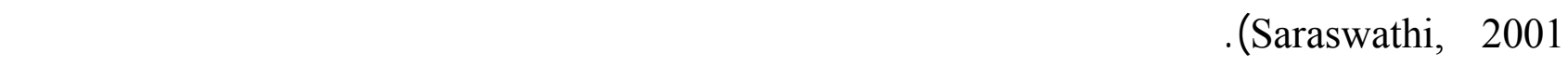

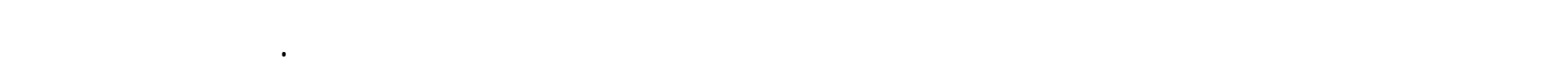

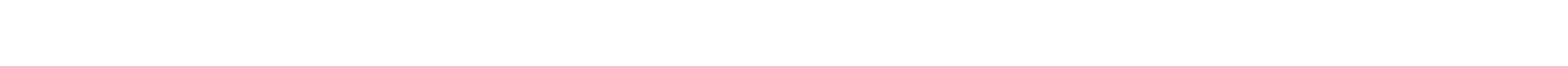
Organic Field Effect (مالترلنزس تورات الع مضوية (OLED) (Organic Light Emitting Diode)

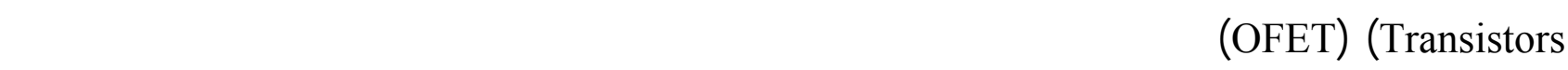

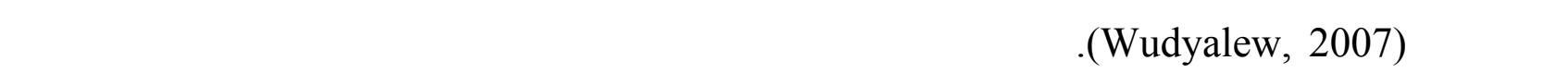

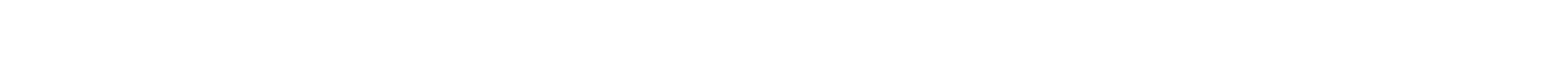

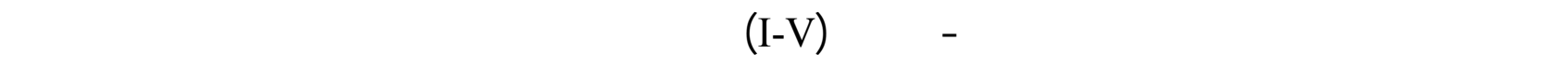

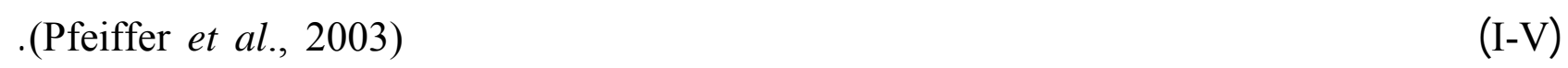

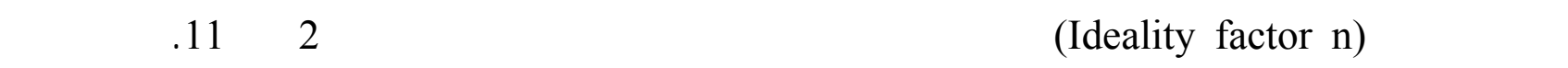

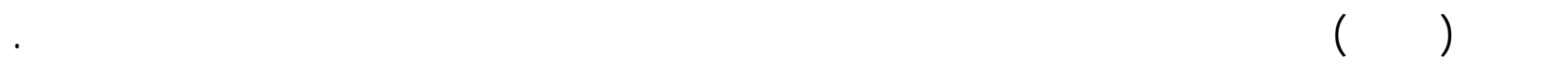

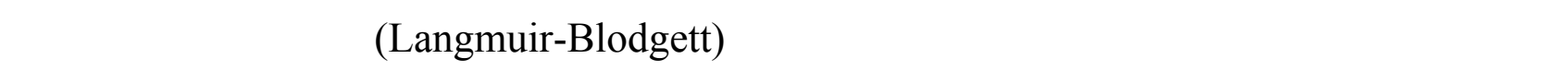

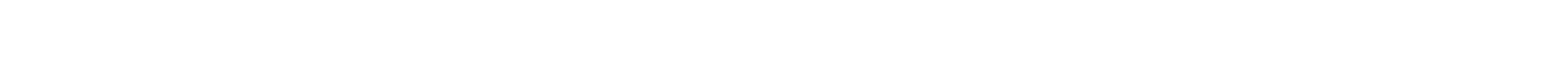

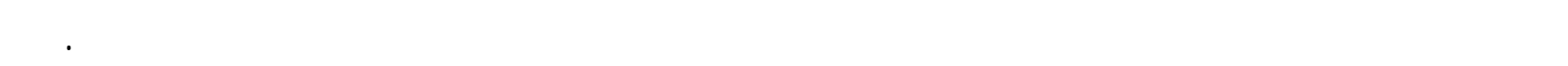

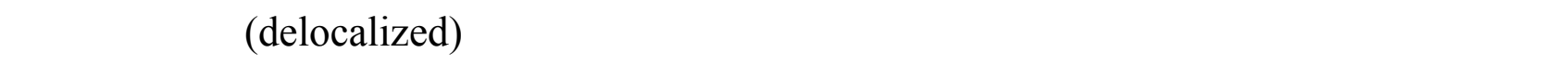
الجزيئت المتجاورة. وبسب هذه القوى الضعيفة فلن الطيف الالكترونية والاهتزازية تربط بتلك الانقالات 
في الحالة الغازية. منذ 1990 جرت المحاولات لصناعة معن -شبه موصل عضوي ثنائيشوتكي وبط -رق تحضير مختلفة لتسيب الغشاء اللاعضوي وقم فمير خواصها الكهربائية (Guirong et al.,2002).

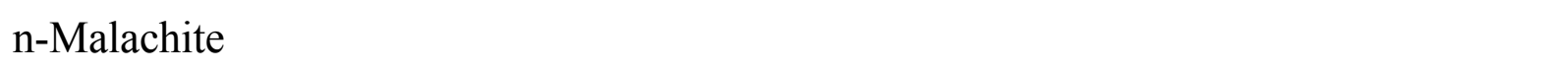
ولستخلمت وريقة I-V green وعلمل المثالية في الهسابت.

\section{الجلب الظاري}

إلّ الخواص الكهربائية لوصلةشوتكي يم تحليلها بافتراض ظارية الانبعلث الحراري لنموذج ح لجزز

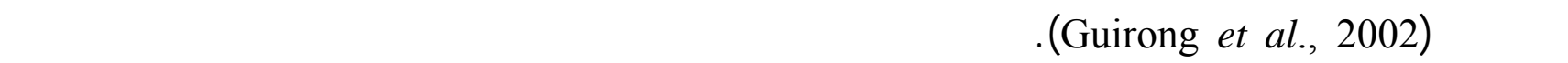
حلملات الشحنة عبر الطقة البينية للنطحية للاتصل، والتيارسيكون دالة لفولتية الانحياز المستخدمة.

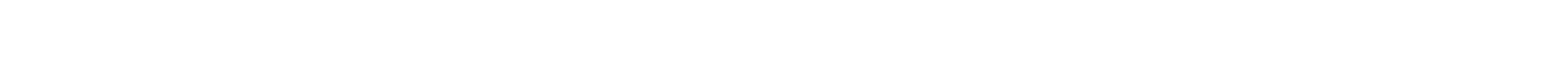

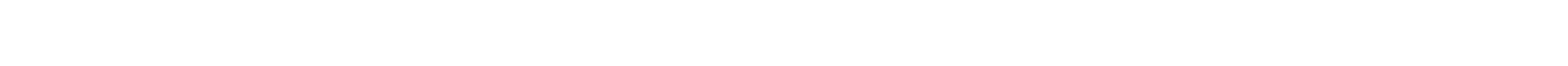

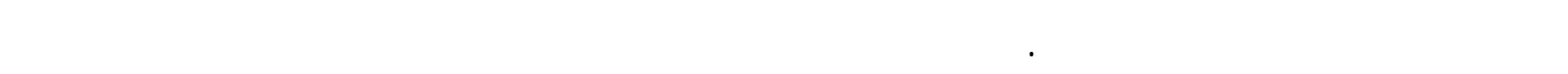

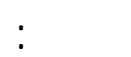

$$
\sigma=\sigma_{0} \exp \left[-\mathrm{E}_{\mathrm{a}} / \mathrm{K}_{\mathrm{B}} \mathrm{T}\right]
$$

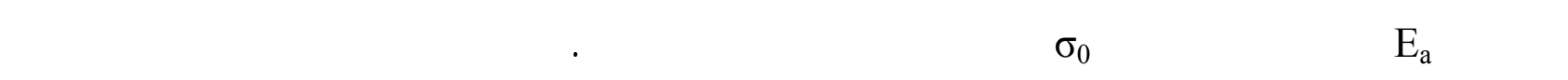

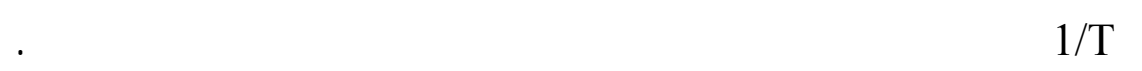

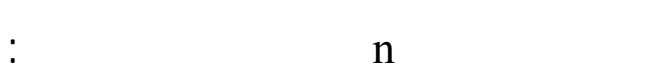

$$
\mathrm{n}=\mathrm{e} / \mathrm{K}_{\mathrm{B}} \mathrm{T}[\partial \mathrm{V} / \partial(\operatorname{lnI})]
$$

من درلسة الحالة المثالية لثنائيشوتكي قم لستنبط معادلة لمسلب ارتفاع حلجز الجهد النبطة وكالت المعادلة .(Williams and Rhoderick,1988) :

$$
\Phi_{\mathrm{Bn}}=\left(\mathrm{K}_{\mathrm{B}} \mathrm{T} / \mathrm{e}\right) \ln \left[\mathrm{A}^{*} \mathrm{~T}^{2} / \mathrm{J}_{\mathrm{sT}}\right]
$$

حيث أن 
ويمكن ليجاد قيمةطاقة التنشط من المعادلة الآتية :

$$
\operatorname{Ln}\left[I_{f} / T^{2}\right]=\ln \left(A_{e} A^{*}\right)-e\left(\Phi_{B n}-V_{f}\right) / K_{B} T
$$

حيث أن

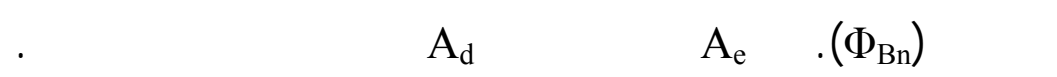

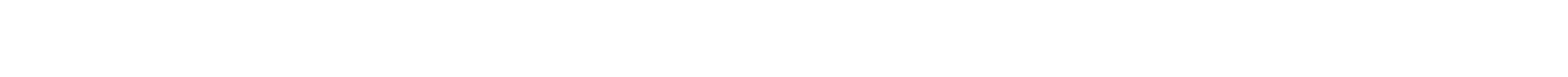

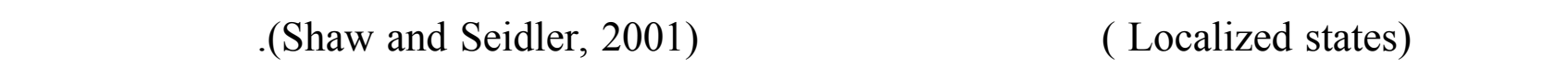
الموصلات اللاعضوية، فلثباه الموصلات العضوية يمكن قعيمها لغرض تغيير توصيليتها.

مق لمتخدلهشبه الموصل العضوي n-Malachite green وص يغته الكيميائي ة هـ مي

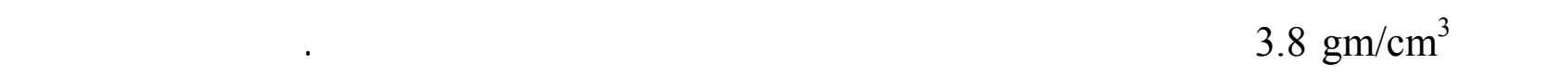

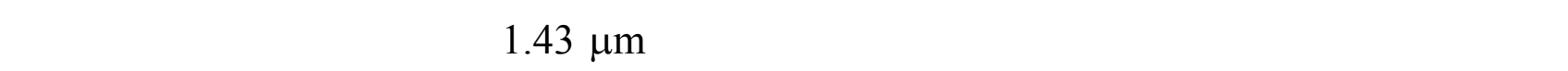

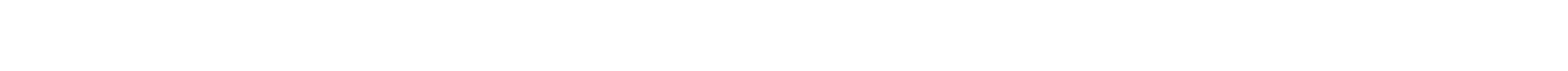
لتريب الغشاء الرقيق العضوي وقم هسلب اللسمك من العلاقة : (Flow Coating Technique)

$$
\mathrm{t}=m / \rho \cdot \mathrm{A}
$$

م : كثافة المادة بوحة

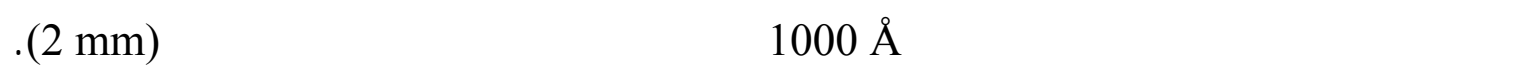

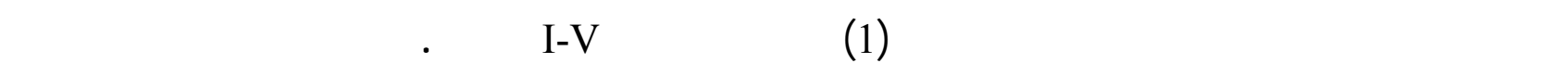

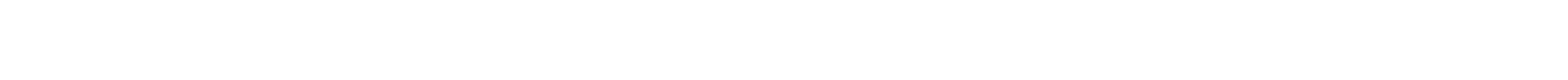
دلخل فرن حراري دائري الفتحة يمكن اللسطرة على درجة حرارة النبطة. 


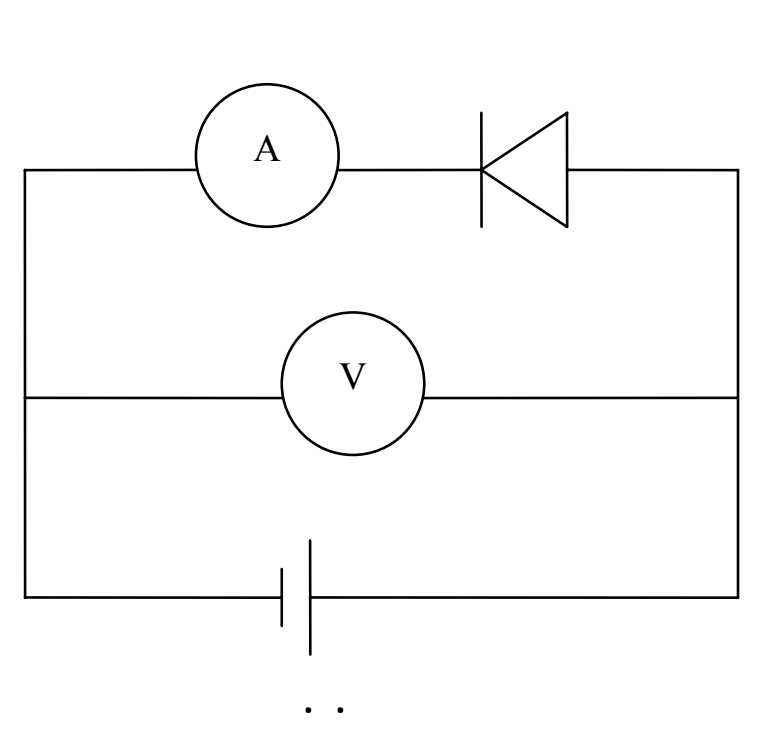

للشكل 1: الدائرة الكهربائية المستخلمة لدرلسة خواص المنحني I-V للنبطة.

\section{النتائ ج والمناdثة}

للشكلين (2) و(3) يوضحان العلاقة بين التيار والفولتية للإنحيازين الأملمي والعكسي عن د درج ـة حرارة الغرفة في حالتي الإضاعة والظلام للثنائي القي. إلّ العلاقة I-V وجدت غير غطي ـة وظه -ر ت أثير

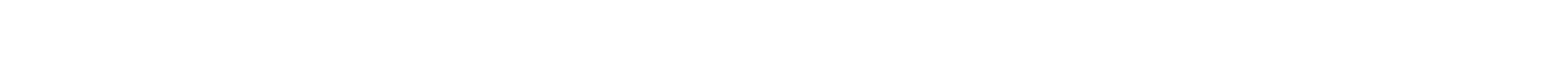
بلستخدلمها خليةشسية، وأنَشحنة التوصل لاتمل فتط بالحلملات الحرة (إلكترون - فجوة) كما في لثث ـبه

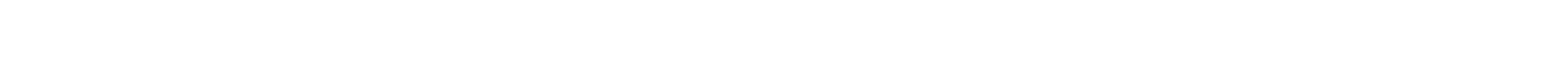
.(Patidar et al., 2006)

لِّلْ خواص I-V لهذا الثنائي توضح تأثير القويم (Rectification) والتي نشير إل م تك -وينطقة لستنزف عند اتصل معنن -شبه موصل، فعند الفولتية التي قيمتها لكبر من ( Volt 1) فإن التي اري يـ صحح

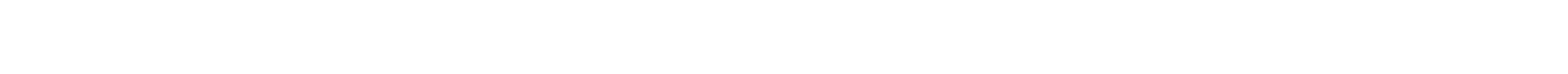




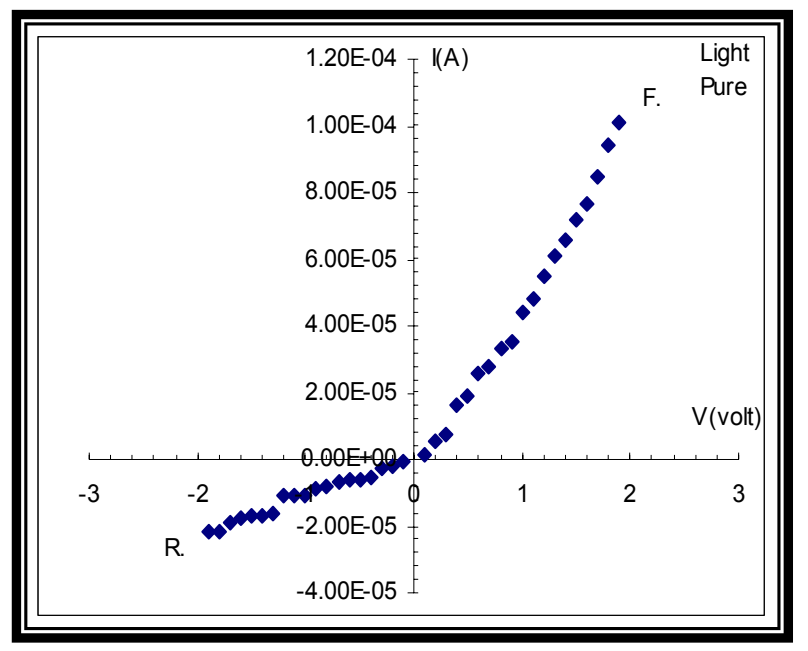

الشكل 2: خواص تيار - فولتية لثنائي A1/ Malachite green/In في حالة الإضاعة.

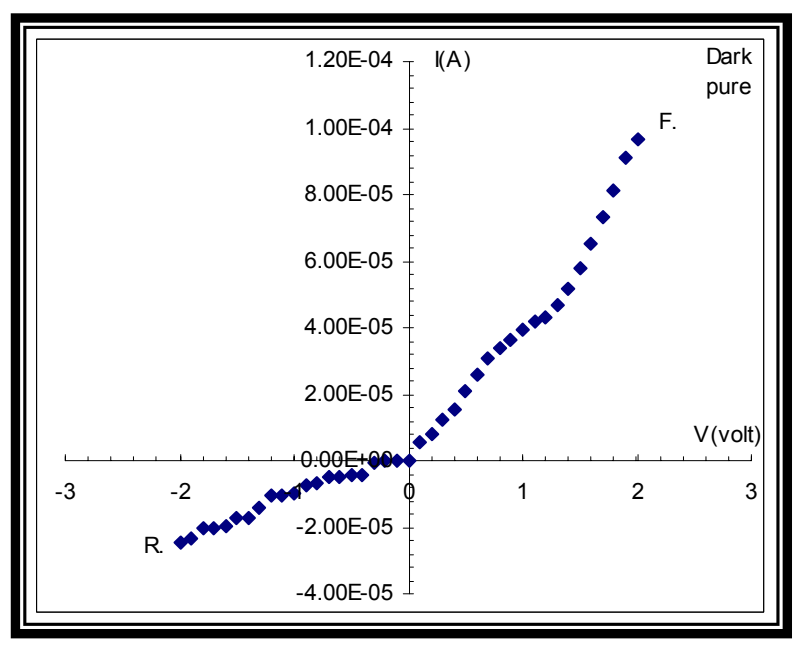

الثشل 3: خواص تيار - فولتية لثنائي Al / Malachite green/In في حالة الظالام.

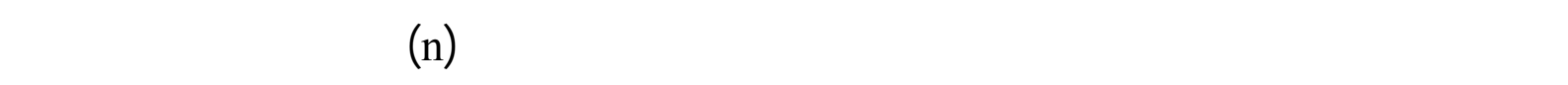

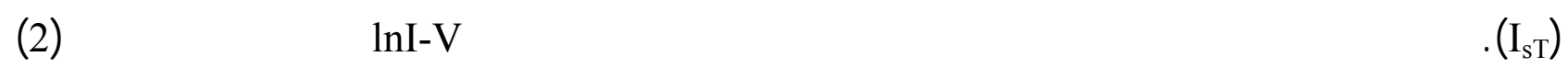

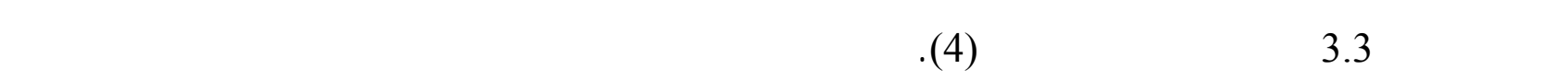
قيمة تيار التشع وكانت 3 X 3 A 


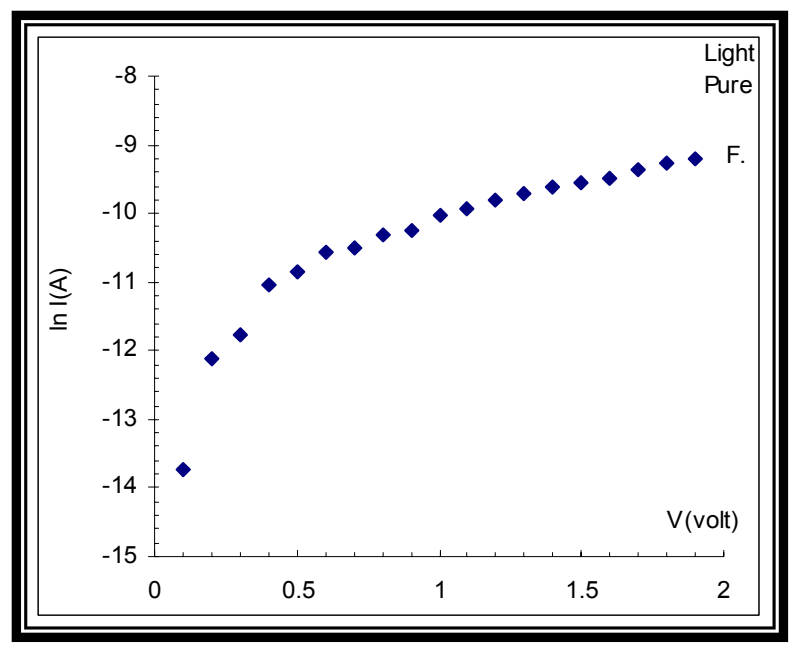

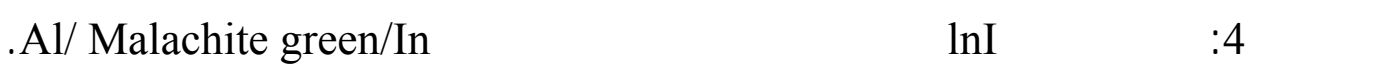

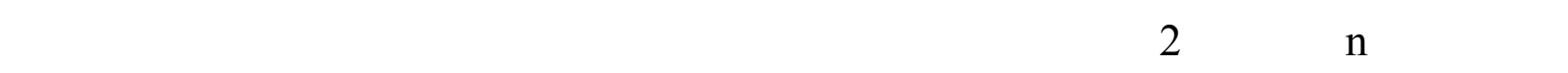
الأوكسيد البينية بين المعن وشبه الموصل العضوي والمقاومة المتوالية، وألّ آلية انتقل التيار هـ مي إء الة إتحاد الحلملات. إلّ المقاومة المتوالية لها معنى في المطقة غير الغطية للعلاقة I-V وال ششل (5) يوض ح ح العلاقة بين dV/dlnI والتيار I . حيث ألهميل الغط المسققيم هو R كما في المعادلة أننه:

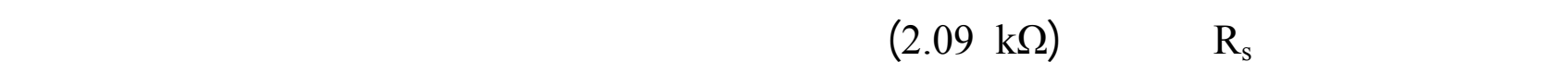
(Caglar et al., 2008)

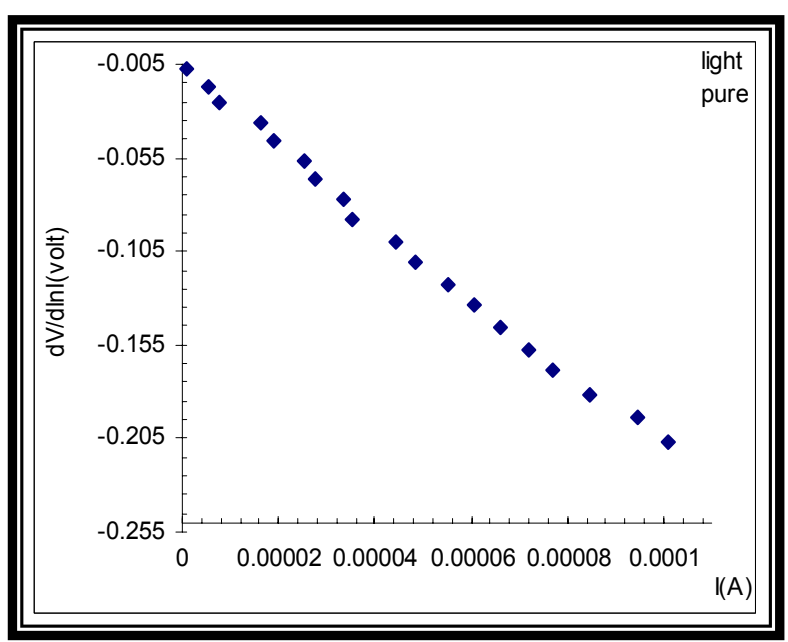

لشكل 5: العلاقة بين dV/dlnI والتيار I لثنائي Al/ Malachite green/In. 
للشكل (6) يبين علاقة إعتماد التوصيلية على درجة الحرارة في حالة الإضاعة حي ث أنَ التوص يلية

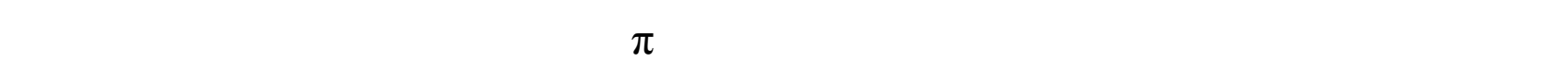

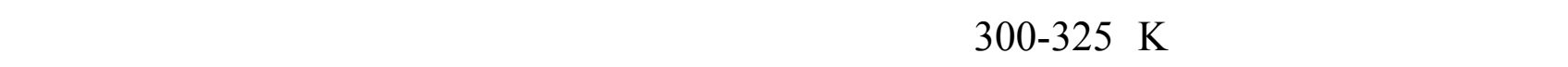

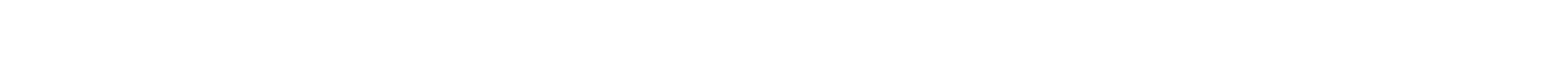

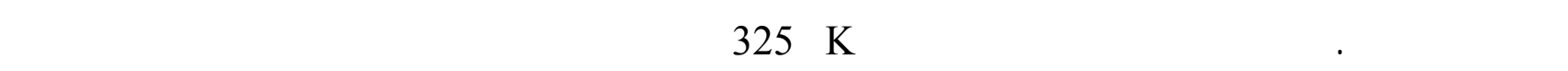

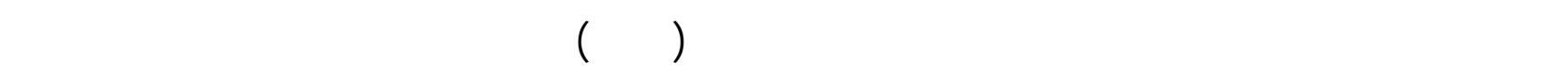
جمي ع الحلملات تستفذ من المرله _ز المتمرك ـزة الم حزمة التوصل (Yakuphanoglu et al., 2004).

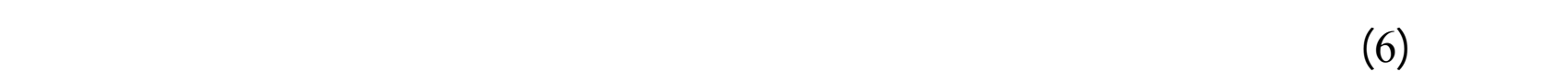

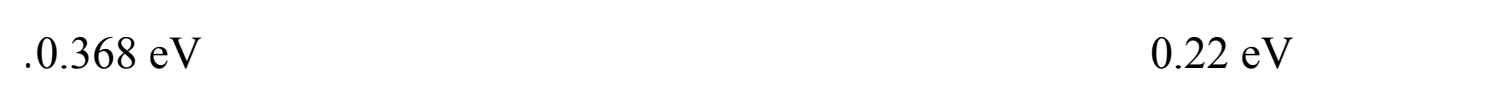

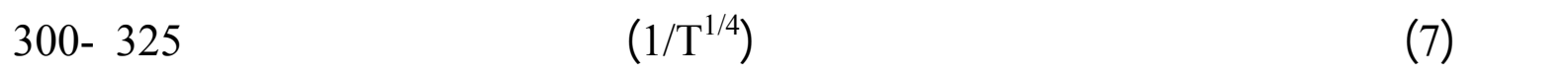
Kيث العلاقة خطية مما يؤكد ان الية القفز(VRH) هي المسؤولة عن التوصيل بالقرب من مستوي فيرمي.

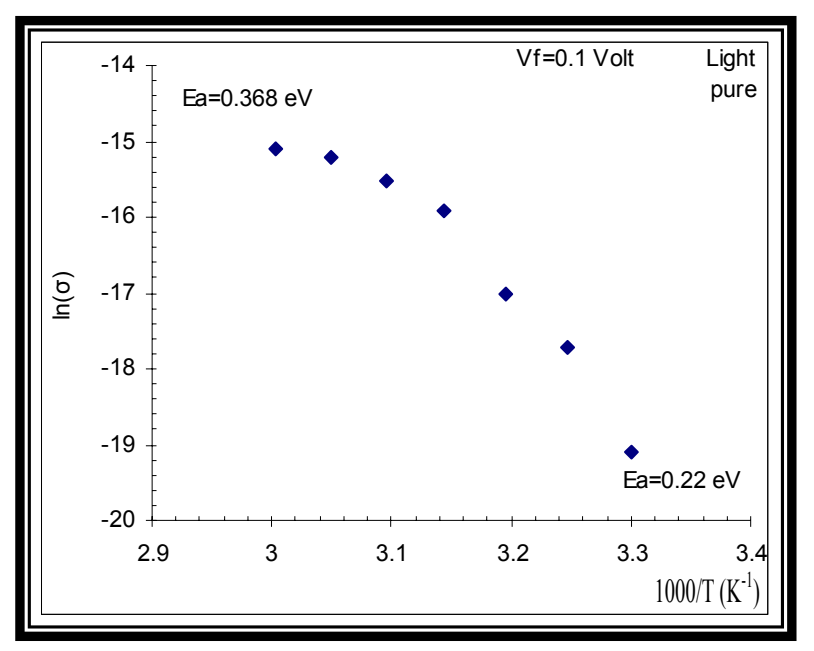

الشكل 6: العلاقة اللوغاريتمية للتوصيلية مع درجة الحرارة لثنائي Al/ Malachite green/In . 


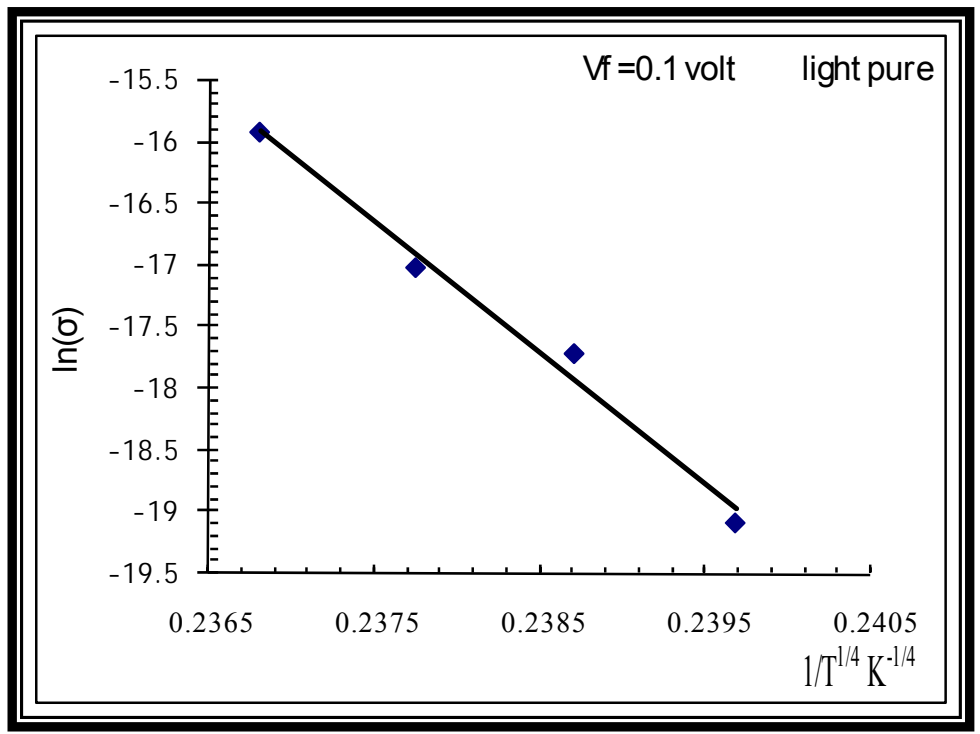

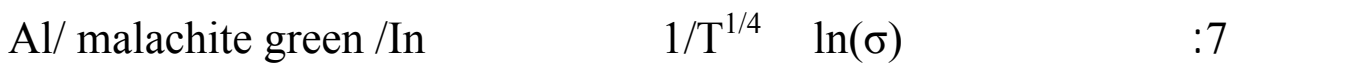

للشكل (8) يبين العلاقة بين (n $\left(I_{f} / T^{2}\right)$ ودرجة الحرارة في حالة الإضاعة حيث يلاقظ تغير ارتق Lاع

حلجز الجهد ( $\Phi_{\text {Bn }}$ مع درجة الحرارة ومنها لمكن الحصول على قيمتين لحلجز الجهد وكانت قيمته 1.71 عند درجة حرارة الغرفة بينما كانت قيمته 1.18eV عند درجلت حرارة أعلى وكانت القيم في ظ ابق مع قيم البلحثلن ( Zubair and Muhammad, 2008 (

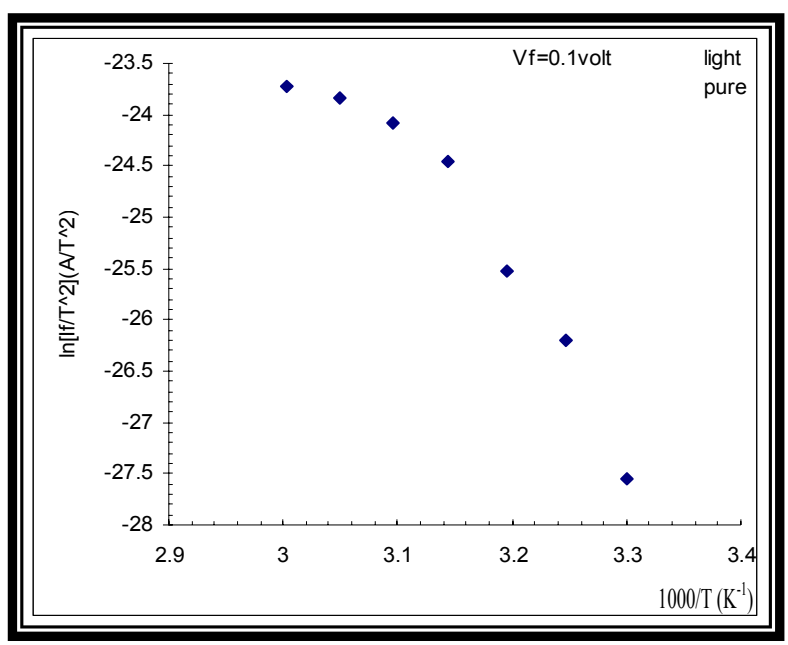

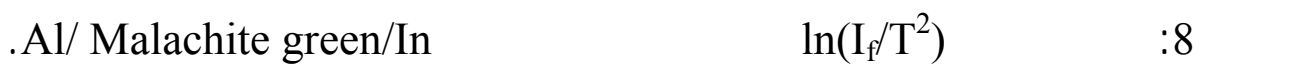




\section{لالستنتلحت}

في ضضوء التنائج التي حصلنا عليها أمكننا لمتتنتاج الآتي :

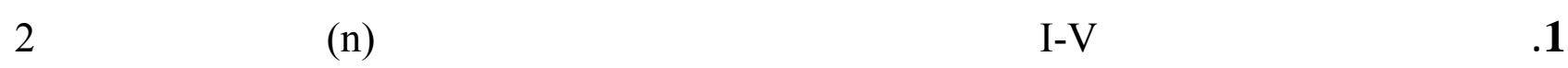

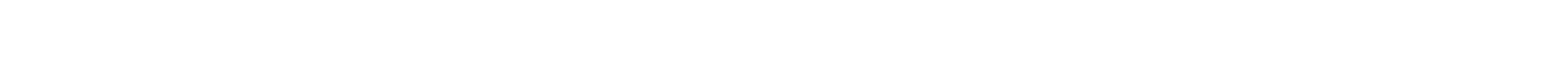
نتنائج بلحثن آخرين (Nicholas and Neliza, 2007) 2. إن قيمة المقاومة المتوالية (R) للنموذج القي ققع ضنمن الحدود المعروفة للأشبل الموصلات العضوية .(Caglar et al., 2008)

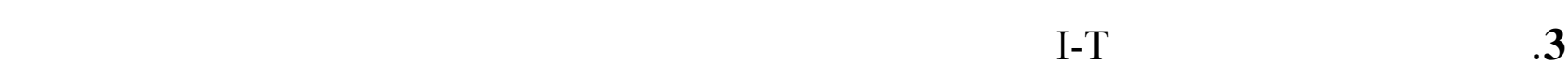

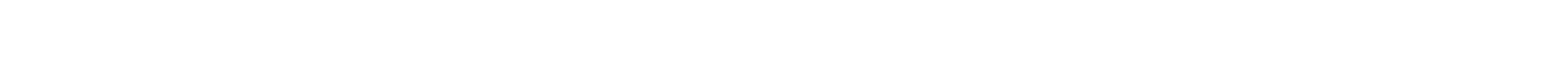
يتوافق مع نتائج بلحثن لخرين (Zubair and Muhammad , 2008).

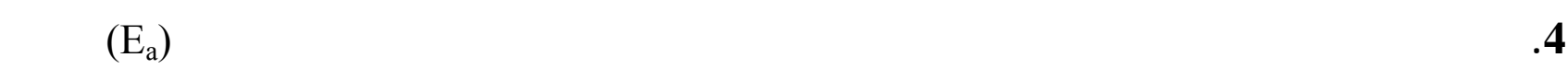

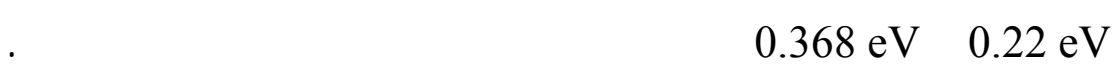

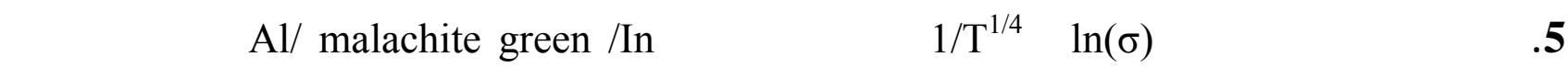
التوصل هي آلية قفز للحلملات (VRH) بالقرب من مستوي فيرهي.

\section{المصاكر لأجنبية}

Caglar, Y.; Yakuphanoglu, F.; Ilican, S., Caglar, M. (2008). "Electrical Characterization of $\mathrm{ZnO} /$ Organic Semiconductor Diode". J. Optoelectronics and Advnced Materials, 10(10), pp. 2584-2587.

Guirong, L.; Tianhong, C.; Kody, V. (2002). "Fabrication and Electrical Characteristics of Polymer-Based Schottky Diode" . 10th. Meeting of the Symposium on Polymers for Microelectronics, Winterthur, Delaware.

Nicholas, P. P. ; Neliza, L. B. , (2007) ." Schottky Diode From A single Polyaniline Nanofiber " , University of Puerto Rico at Humacao, Department of Physics and Electronics, Fisi $4192-001$.

Patidar, D.; Jain, N.; Saxena, N.S.; Sharma, T.P.; Kananbala, Sharma (2006). Electrical properties of CdS/Polyaniline hetrojunction. Brazilian J. Physics, 36(4A), 106.

Pfeiffer, M.; Leo, K.; Zhou, X.; Huang, J.S. (2003). Doped organic semiconductor : physics and application in light emitting diode. Organic Electronics. 4, $89-103$.

Shaw,J.M ; Seidler, P.F.(2001). Organic electronics: Introduction, IBM, Res. and Dev. 45, (1).

Syed, A. P.; Saraswathi, R. (2001). Junction Properties of Metal/Polypyrrole Schottky Barriers. J. Applied Polymer Sci . 81, 2127-2135. 
Syed, A. P. ; Saraswathi, R. (2000). Junction Properties of Metal/Polypyrrole Schottky Barriers". Department of Materials Science, Madurai Kamaraj University, Madurai 625021 , Tamilnadu , India.

Williams, R.H. ; Rhoderick, E.H. (1988). "Metal - Semiconductor Contacts ", 2nd. Edn. , Oxford University Press . New York, Toronto.

Wudyalew, W. (2007). Electrical Transport and The Schottky Effect In Organic Nanoelectronic Materials and Devices. M.Sc thesis, New Mexico State University Las Cruces, New Mexico.

yakuphanoglu, F. ; Aydin, M.; Arsu, N. ; Sekerci, M. (2004). "A Small-Molecule Organic Semiconductor" .Фn3nka n ТехНnka полуПроВоДНnkoB,2004,ТОМ 38,ВblП.4

Zubair, A. ; Muhammad, H. S. (2008). Investigation of the electronic properties of (au/methyl - red/ag) surface type schottky diode by current - voltage method. Proceedings of World Academy of Science, Engineering and Technology. 33, 2070 -3740 . 